\title{
Current Situations and Challenges of Occupational Disease Prevention and Control in China
}

\author{
Huanqiang WANG ${ }^{1}$ and $\mathrm{Li} \mathrm{TAO}^{1}{ }^{*}$ \\ ${ }^{1}$ National Institute of occupational Health and Poison Control, Chinese Center for Disease Control and \\ Prevention, Nanwei Road No.29, Xicheng District of Beijing, 100050, China
}

Received December 5, 2011 and accepted January 6, 2012

\begin{abstract}
China is one of ancient countries with nearly 5,000 yr history of civilization, with land area of 9,600,000 km², including 4 municipalities, 23 provinces, 5 autonomous regions and 2 special administrative regions. There are 56 nations and 1.37 billion peoples (2010, November) ${ }^{1}$. In 2010, GDP was 40120.2 billion Yuan, and per capita GDP was 3,600 dollars. Employment population nearly reached 780 million peoples, including a total of 226 million migrant workers ${ }^{2}$. At present, China is in the rapid development period of industrialization, urbanization and globalization, and workers face the traditional and novel dual occupational hazards. Also, prevention and control of occupational disease faces severe situations and challenges.
\end{abstract}

Key words: Occupational health, Occupational hazards, Occupational disease, Prevention and control, Current situations and challenges, Migrant workers

\section{Achieved Basic Achievements}

In recent years, the Chinese government adheres to the construction of harmonious society and the peopleoriented concept. In addition, the government attaches great importance to workers' occupational health and every years continuously invests 100 million Yuan (about equivalent to 15.8 million US Dollar) to take a series of effective measures annually for supporting the prevention and control of occupational diseases. Also, prevention and control work of occupational diseases has obtained an apparent progress.

Firstly, primarily established the supported occupational hygiene regulations, standards and technical specification system of Law on Prevention and Control of Occupational Disease of PRC. In 2002, National People's Congress Standing Committee of china issued Law on Prevention and Control of Occupational Diseases, and the Ministry of

*To whom correspondence should be addressed.

E-mail: niohplt@sina.com

(C)2012 National Institute of Occupational Safety and Health
Health prepared and issued 17 supported regulations associated with Law on Prevention and Control of Occupational Disease, and prepared and revised 667 occupational health standards. According to the main occupational disease hazard, it still prepares a large amount of occupational health technology specifications and primarily established the law and regulation and the standard system of occupational disease prevention and control. See Fig.1.

Secondly, stand out emphases and enhance supervision and law-enforcement strength of occupational disease prevention and control. Since 2002, the relevant departments of the state have launched special rectification activity of occupational hazard for many times, and supervision departments annually carry out important occupational health supervision and inspection for industries and populations with obvious occupational hazards.

Thirdly, enhance the supervision of occupational hazards in construction projects, and control of occupational diseases from the sources. The relevant departments of the state prepared Classification Management Methods for Occupational Hazards in Construction Projects, Construc- 


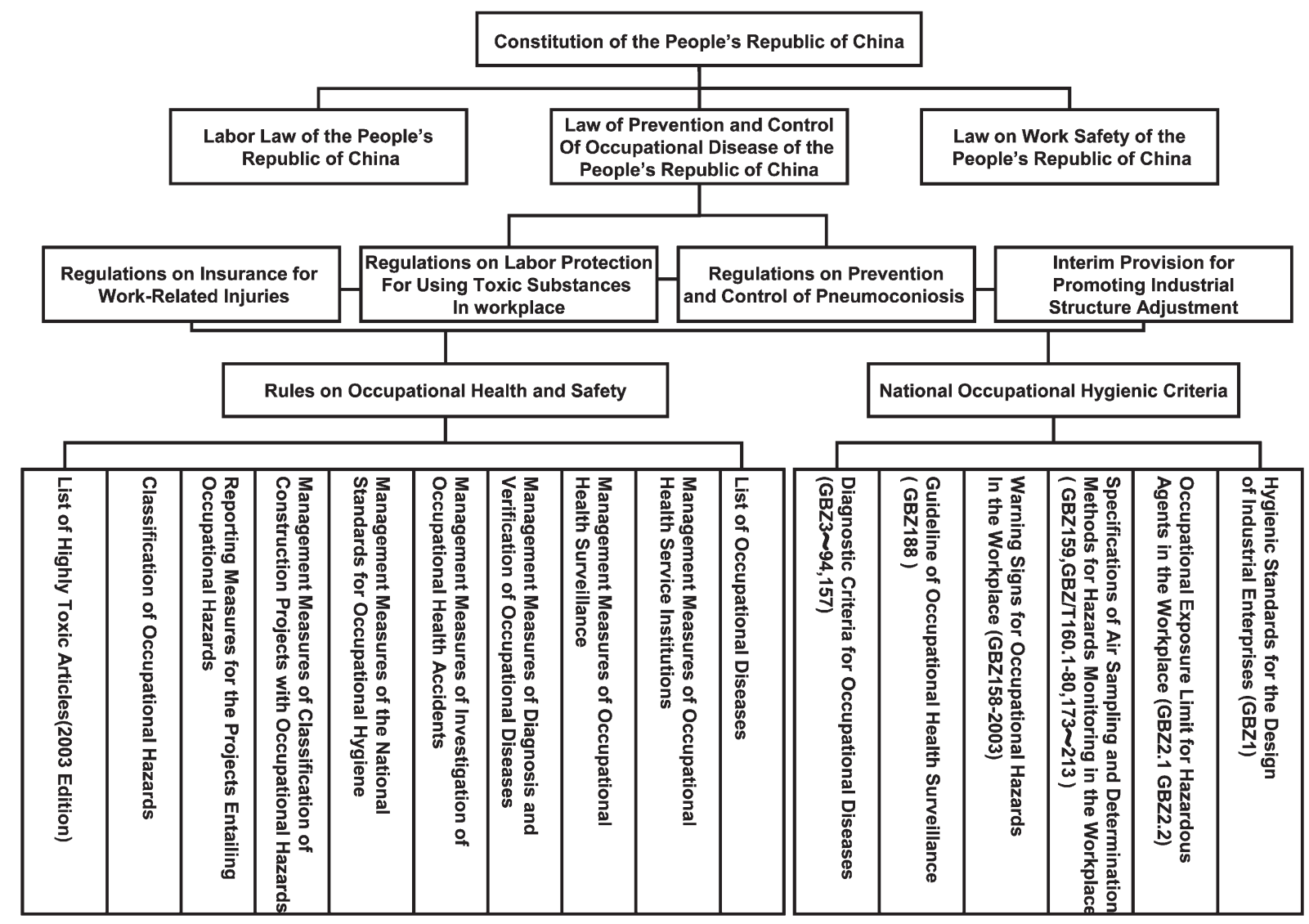

Fig. 1. Legal frameworks of occupational health in China.

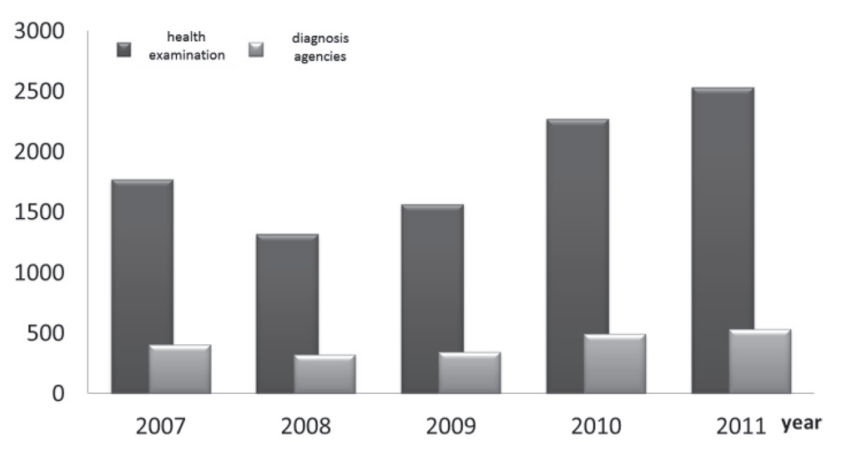

Fig. 2. The number of occupational health examination and occupational diseases diagnosis agencies.

tion Project Occupational Health Review and Approval Provision, etc. and strengthen and specify the construction project occupational health supervision. Also, the supervision strength of construction project occupational health review is increased step by step.

Fourthly, strengthen supervision of occupational health agencies and constantly improve the occupational health technical service ability and level. According to the relevant provisions of Law on Prevention and Control of Occupa-

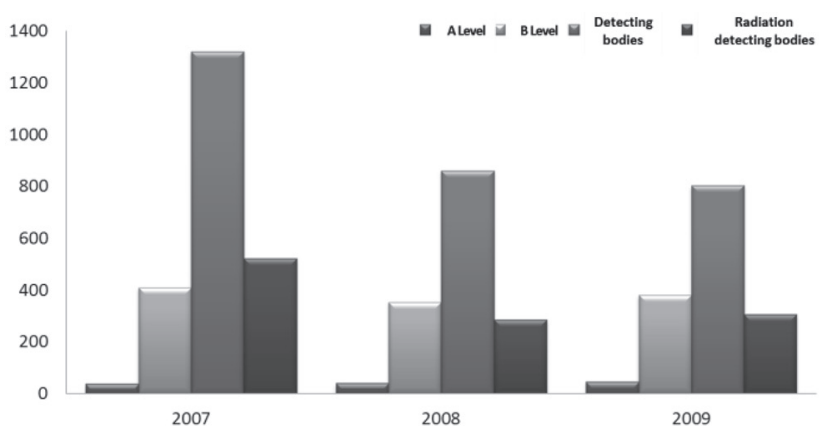

Fig. 3. Developing of occupational health service.

tional Disease and the licensing system of occupational health service agencies, China has approved respectively 40 A level and 420 B level construction project occupational hazard evaluation agencies, 25 chemicals toxic identification agencies, 9 detection qualification agencies of protective equipment and radioactive products, 1,467 occupational health surveillance agencies and 397 occupational disease diagnosis agencies at present. See Figs. 2 and 3.

Fifthly, organize to carry out basic occupational health 


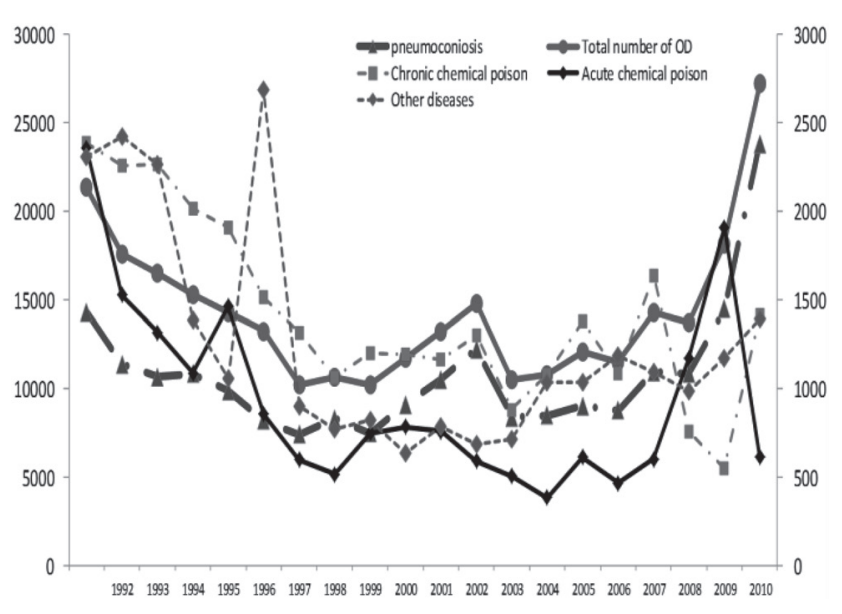

Fig. 4. Nosogenic status of occupational disease in China.

service (BHOS) pilot activity to strive to improve the occupational health service ability and accessibility. In August, 2006, the Ministry of health selected the 19 counties (districts) in 10 provinces with concentrated labor inflow and outflow to carry out the BOHS pilot work for exploring the primary occupational health service model and expanding the occupational health care coverage. The pilot work have obtains initial effectiveness. At present, it has expanded to 65 counties (districts) in 29 provinces (area).

Sixthly, strengthen publicity and education to improve the social law awareness of occupational disease prevention and control. From 2002, the Ministry of health determines the last week of April each year as the publicity week of the Law on Prevention and Control of Occupational Disease and annually determines a theme and uniformly prepares promotional materials for carrying out the relevant publicity work. The working situation of the whole society concerning the prevention and control of occupational diseases has been primarily formed by several-years constant publicity and education for Law on Prevention and Control of Occupational Disease.

\section{Nosogenic Status and Epidemic Characteristics of Occupational Diseases}

Although, prevention and control of occupational disease in china has made a remarkable progress, it is still facing many challenges as China is in the primary stage of socialism, productivity level isn't high, differences between cities and between regions are too large, the social economic development is very uneven, and the law

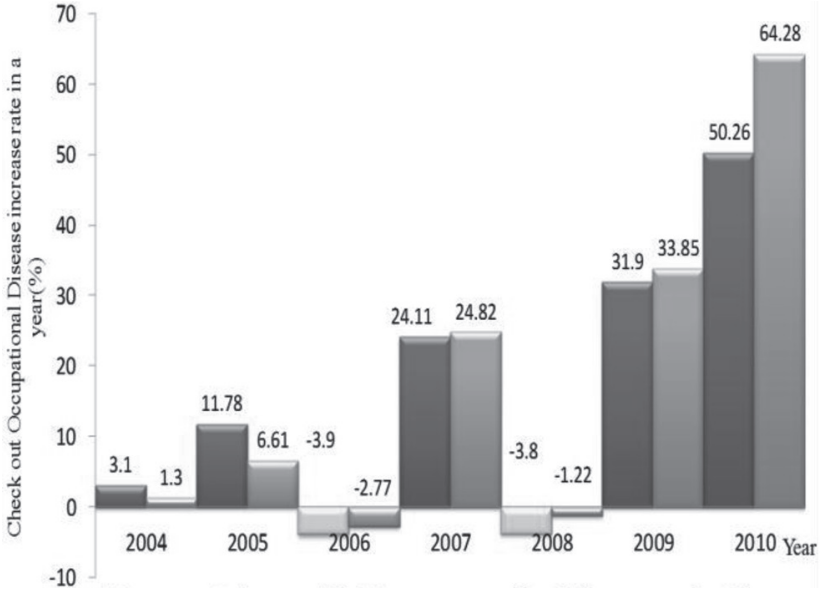

Fig. 5. Increase rate in a year (\%) of the new occupational disease cases in China.

awareness of employer for the prevention and control of occupational diseases isn't high.

1. Morbidity of occupational diseases is very high, and the newly-reported occupational disease cases are rising year by year. By the end of 2010, a total of occupational diseases reached 750,000 cases, including 653,000 cases of pneumoconiosis and 47,000 cases of occupational poisoning. In 2010, newly-reported occupational diseases reached 27,240 cases, increasing by 50\% compared with 2009. The reported pneumoconiosis reached 23,812 cases, increasing by 64\% compared with 2009. See Figs. 4 and 5 .

However, the coverage level of occupational health surveillance in china is still very low. It is about $30 \%$ for state owned enterprises and foreign-investment enterprises, and it is about 3\% for small and medium-sized enterprises. The coverage level of occupational health surveillance was estimated less than $10 \%$. It can be said that patient with occupational diseases found by occupational health surveillance every year are very few. In addition, some concealed and non-reported problems also influence the actual morbidity data of occupational disease.

2. Occurrence of group events related to occupational disease is becoming the prominent public health problem influencing the social harmony and stability. With the diversification of enterprise ownership forms and urbanization and industrialization of the rapid economic development, Chinese labor system is more flexible, and employment appears apparent mobility and temporary. Especially, working of migrant workers has some characteristics of group migrant and relative aggregation employ- 


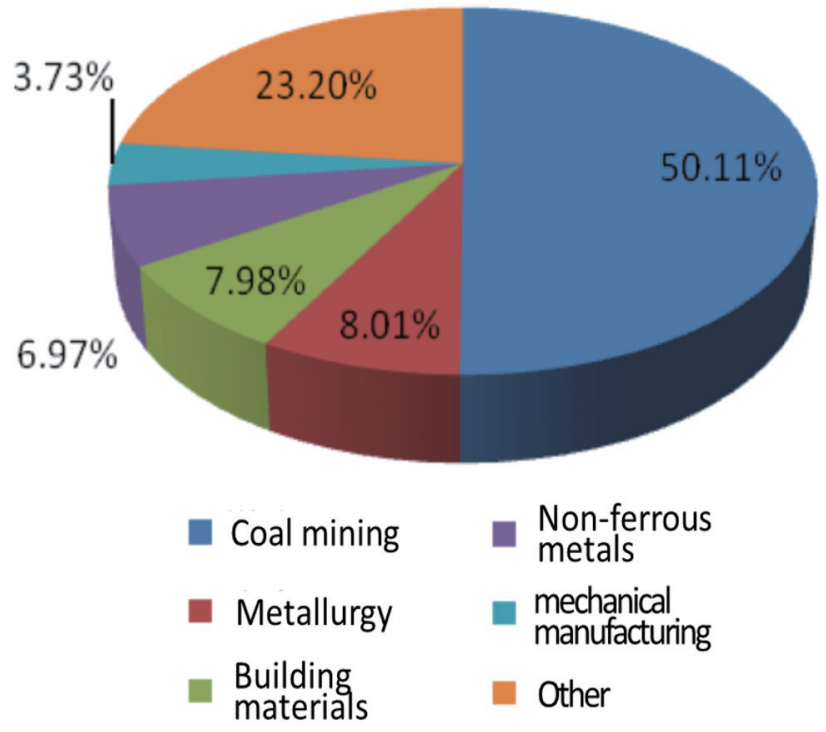

Fig. 6. Top 10 industries of incidence of pneumoconiosis in the past $10 \mathrm{yr}$.

ment. Therefore, it has become a notable characteristic of occupational disease occurrence that groups suffer from occupational diseases, and it has become the prominent public health problem of influencing the social harmony and stability. For example, for Suzhou Lianjian Technology Company mainly producing medium and small size LCD for Apple Company, more than 100 workers with nhexane poisoning are caused due to the illegal use of hexane solvent instead of alcohol for wiping mobile phone.

3. Occupational diseases have become the important factor restricting the sustainable development of social economy. The economic loss induced to occupational diseases is very serious because of high morbidity of occupational disease. According to the relevant studies at end of 90's in the last century, the average annual economic loss of each case of patient with pneumoconiosis reached about 3.41 ten thousands Yuan. The occupational disease statistics report in recent 10 yr shows that the average age of the onset of pneumoconiosis is $19.39 \mathrm{yr}$, and the average age of death is 73.2 yr. If a labor aged 38-40 yr suffers from pneumoconiosis, he can living for $30 \mathrm{yr}$ after treatment, and the direct economic loss can reach 1 million Yuan. Chinese occupational safety and Health Association estimates that annual direct economic loss due to occupational diseases and industrial injury accidents in china reaches 100 billion Yuan, and the indirect economic loss reaches 200 billion Yuan ${ }^{3)}$.

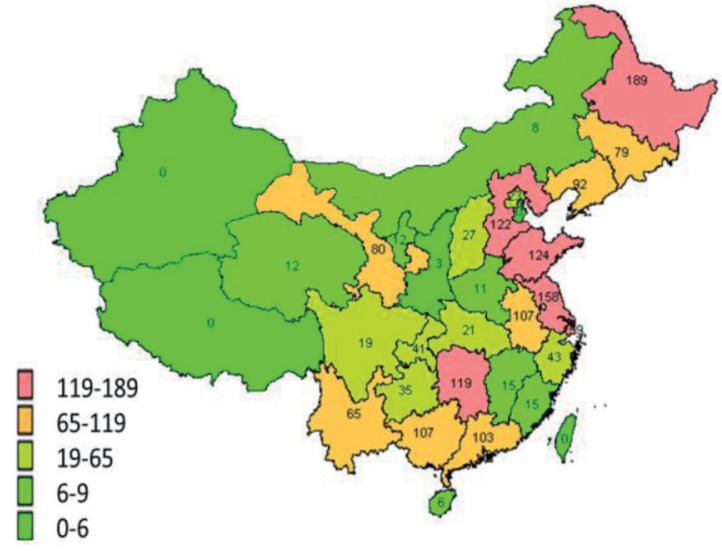

Fig. 7. Regional distribution of acute occupational poisoning between 2006-2008.

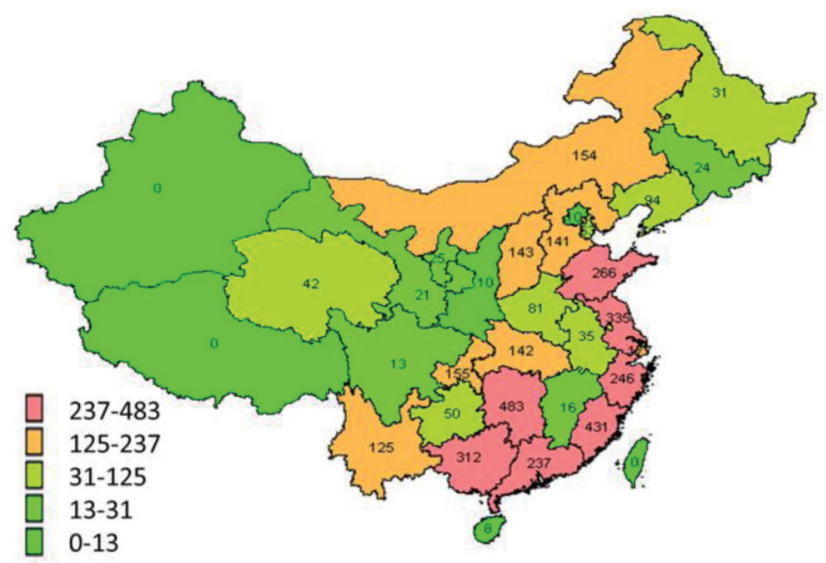

Fig. 8. Regional distribution of chronic occupational poisoning between 2006-2008.

4. Distribution by types of occupational diseases showed that obvious industrial feature. Figure 6 showed the pneumoconiosis mainly occurs in coal mining, metallurgy, building materials, non-ferrous metals and mechanical manufacturing industry. Among pneumoconiosis, more than half of patients were coal workers with pneumoconiosis, and they accounted for $50.11 \%$ of pneumoconiosis patients, and silicosis patients accounted for $30 \%$. Also figure showed the acute occupational poisoning mainly happens to chemical, building, coal mining, light industry and non-ferrous metal industry, Chronic occupational poisoning mainly happens to non-ferrous metal, light industry, chemical, mechanical manufacturing and metallurgy industry.

5. Regional Distribution of occupational diseases 
showed some characteristics.

In the eastern coastal areas, major occupational diseases are chronic occupational poisoning, such as poisoning caused by lead or cadmium and leukemia caused by exposure to benzene. While in northeast China's old industrial base and central China frequently happens to acute poisoning.

6. Pneumoconiosis and common acute and chronic occupational poisoning are the most serious occupational diseases. Health supervision bulletin showed that the workers engaged in dust operations constitute the major population exposed to occupational hazards and the percent of workers exposed to dust hazards among the workers with occupational hazard exposure was $45.78 \%$ in china ${ }^{4}$. In recent years, the new pneumoconiosis cases there are a fast growth. According to the national occupational diseases report, reported pneumoconiosis patients obvious increased annually. In 2010, pneumoconiosis patients increased to 24,000 from 18,000 cases in 2009. In addition, national occupational disease report showed that the number of pneumoconiosis patients accounted for $2 / 3$ the total number of patients with occupational diseases. Acute and chronic occupational poisoning cases accounted for 19 per cent of all occupational diseases. Therefore, pneumoconiosis and occupational poisoning are primary occupational disease and main point to prevent and control in China.

\section{Challenge of Prevention and Control of Occupational Disease in China}

1. A large part of Chinese workers were exposed to occupational hazard, and the task of prevention and control for occupational disease is difficult. The second national economic census results show that by the end of 2008 , there were altogether 1.903 million industrial enterprise by legal person units and 117.383 million employees in the whole country ${ }^{5}$. Accordingly, it was speculated that workers exposed to occupational hazards in industrial enterprise nearly reached 60-70 million. According to some surveys, the percent of workers exposed to occupational hazards in the whole workers was about $40 \%$. Therefore, it was speculated that laborers exposed to occupational hazard factors were possibly about 150 million.

2. Occupational hazard spreading more than 30 industries. According to the occupational disease report in recent $10 \mathrm{yr}$, there are reported cases of occupational diseases in more than 30 industries, such as coal and mine exploration, metallurgy and non-ferrous metal smelting, chemical industry, architecture, machinery manufacturing, agriculture, forestry, wood processing, leather manufacturing, luggage processing, footwear, automobile manufacturing, precious stones processing, waste recycling, container manufacturing, computer, medicine, biological engineering, etc.

3. Migrant workers become the main population of occupational hazard exposure.

According to the statistics, by the end of 2010, there were 242 million migrant workers in China ${ }^{2)}$. Because of the household registration system and the employment system connected with the household registration system, migrant workers are squeezed into secondary labor market with low income, poor working conditions, low pay and poor welfare in the oversupply of labor market. Their employment structures mainly include some industries with low level and heavy physical force such as architecture, industry and service industry so $\mathrm{on}^{6,7}$. Their work mainly concentrates in small and medium-sized enterprises of intensive-labor industries such as mining industry, architecture, machinery manufacturing, etc. In these industries, safety production risk is high, and the occupational hazard exposure is relatively common. Casualties due to safety production accidents are more, as migrant workers become the main composition of labor force, they have become the main population of exposure to occupational hazard. For example, a total of 1,001 migrant workers from Mashan County in Guangxi went to work in gold mines from 1985 to 1991. Among 600 peoples receiving silicosis disease examination, there were 225 patients with diagnosed silicosis disease. Data from Ministry of national human resource and social security showed that in 2008, among near 1 million persons of industrial injury identification, migrant workers accounted for more than $80 \%$.

Occupational health survey on the second-generation migrant workers aged less than $30 \mathrm{yr}$ in 2010 showed that among nearly 0.1 billion new-generation migrant workers in China, about $60 \%$ of migrant workers worked in the industries with high occupational health risk. On one hand, working environment was generally poor, and on the other hand, working usually was overtime. Near 35\% new-generation migrant workers worked for more than 8 h every day, and more than $66 \%$ migrant workers worked for more than $5 \mathrm{~d}$ per week.

4. The spread of occupational diseases towards rural and 
poor regions is very severe.

Statistical analysis shows that except for Tibet Autonomous Region, there are reported occupational disease cases in other regions of China. As open to the outside world and introduction of foreign capital and advanced technology, some technologies and products with high occupational hazard risk transfer from overseas to domestic, rapidly transfer from cities and industrial regions to countries, transfer from developed regions to undeveloped regions, and transfer from large and medium-sized enterprises to small and medium sized enterprise. In recent years, with the open of eastern regions, development of western regions, rapid growth of middle regions and the revitalization of northeast old industrial bases, occupational hazards obviously trend to transfer from the eastern coastal regions to central and western regions in the capital attraction of mid-west and northeast regions. According to the survey of $\mathrm{MOH}$ in late 1990s, about $37.2 \%$ of foreign-invested and Hong Kong-, Macau-, or Taiwan-invested enterprises involved operations with severe occupational disease risks, and about 34.7 per cent of workers working in these enterprises were engaged in poisonous and harmful operations. About $83 \%$ of township industrial enterprises involved various degrees of occupational hazard, nearly $34 \%$ of workers were engaged in dust and poisonous operations. The incidence rate of suspected occupational diseases was as high as 15.78 per cent.

5. The traditional occupational hazards and the emerging occupational hazards coexist. While pneumoconiosis and chemical poisoning haven't yet been fully controlled, new occupational diseases constantly appear. For example, pneumoconiosis of workers engaging in rush and precious stone processing, hexane poisoning of luggage processing and shoe-making workers and trichloroethylene poisoning and trichloroethane poisoning of electronic workers occur successively in recent years, suggesting that the new occupational hazard is becoming the important factor influencing health of labors. In addition, other occupational issue, such as occupational exposure of the Health Care Workers, safe nanotechnology in the workplace and ergonomics and so on also are need to urgently address the problem.

\section{The Tasks of Prevention and Control of Occupational Diseases in China}

1. Accelerating amendment of the Law on Prevention and Control of Occupational Diseases and improving the legal system.
With the rapid development of society and economy, Law on Prevention and Control of Occupational Disease prepared before $10 \mathrm{yr}$ can't be applicable to the requirements of the prevention and control of occupational diseases, it is necessary to revise by combining workplace occupational health supervision adjustment and the actual requirements of occupational diseases prevention and control. At present, National People's Congress Standing Committee of China is discussing Law on Prevention and Control of Occupational Disease (bill of amendment). Once amended Law is issued, the important task of further perfecting legal system construction will be faced.

2. Actively implement the National Planning of Occupational Diseases Prevention and Control (2009 2015).

In 2009, the State Council issued the National Planning of Occupational Diseases Prevention and Control $(2009 \sim 2015)^{8}$ ), taking occupational disease as a major disease for management. Local governments at all levels must prepare prevention and control planning of occupational diseases of their own regions according to National Planning of Occupational Diseases Prevention and Control (2009 2015) and unify planning, make overall arrangement, strengthen a leadership and establish the sound responsibility system of occupational disease prevention and control to form the supervision chain of occupational disease prevention and control.

3. Enhance supervision strength and urge employers to implement the responsibility of occupational disease prevention and control.

On October 8, 2010, the Central Office issued "Notice as regards Responsibility Division of Occupational Health Supervision Department" (Central Office Issue [2010]104) to determine the new occupational health supervision system. Supervision departments must truly implement the supervision responsibility of occupational disease prevention and control, truly strengthen administrative supervision for occupational disease prevention and control and urge employers to truly implement the responsibility of occupational disease prevention and control according to the responsibility division of Central Office Issue [2010]104 document.

4. Strengthen the construction of occupational disease prevention and control agencies.

Since 2008, the Ministry of Health has issued the relevant documents successively to ask local departments to strengthen the construction of occupational disease 
prevention and control agencies by catching the chance of deepening reform of medical and health system. At the same time, clinical key specialty construction of national occupational diseases, sentinel surveillance of occupational diseases and diagnosis of key occupational diseases and skill training of occupational health examination inspection physicians have been strengthened. Also, constructions of partial city-level and county-level independent occupational disease prevention and control colleges (institutes) have been strengthened. In addition, it is necessary to improve the occupational health service ability on this basis.

5. Strengthen the surveillance of main occupational diseases.

Further strengthen the construction of occupational disease surveillance information platform, carry out the initiative occupational disease sentinel surveillance, strengthen the surveillance, report and management of occupational disease and occupational health information and establish and perfect the surveillance, report and management networks of occupational disease and occupational health information to collect and analyze dynamic information of occupational diseases in time in order to provide theoretical data for preparing the guideline and policy of occupational disease prevention and control.

6. Strengthen scientific researches of occupational disease prevention and control.

With the key fields of occupational disease prevention and control in the national medium- and long-term science and technology development compendium and its priority theme as leadership, the occurrence and development, diagnosis and treatment, emergency rescue and key technology of prevention and control of pneumoconiosis, occupational poisoning, occupational tumor as sally port, dust, gas, radiation, noise and vibration protection technologies and sampling, testing, alarm, respiratory protection and emergency rescue of dust, poison, physical factor and other harmful factors as emphasis, strengthen researched on the key technology of occupational disease prevention and control. At the same time, it still necessary strengthen researches on new occupational hazards possibly caused by occupational stress, fatigue, ergonomics, musculoskeletal disorders, biotechnology, nanotechnology, etc. for adapting to the application development of new technologies, new materials and new technology and truly protecting the health of laborers.

\section{References}

1) China Statistical Yearbooks. China Statistics Press, Beijing. EDITOR'S NOTESEDITORIAL BOARD AND EDITORIAL STAFF New Statistical Yearbooks Published by Statistics Press.

2) Xinhua daily traffic: number of migrant workers in China reached 226 million last year. August 29, 2008. http://www. news.xinhuanet.com.

3) China occupational safety and Health Association: survey on situations and problems of pneumoconiosis, October 2005.

4) Office of the Ministry of health: national health inspection's briefing in 2008, health supervision (2009) 74 .

5) National statistics Bureau of China: Statistical bulletin of national economic and social development in 2010 [1], February 28, 2011.

6) Yang Zhiming: health of migrant workers is an important task of the social security, protecting migrant workers' health forum, Beijing, April 24, 2009.

7) State Council Research Office: research report of China's migrant workers, China real press, April 2006 First Edition first printing.

8) National statistics Bureau of China: Statistical bulletin of national economic and social development in 2010 [1], February 28, 2011. 